\title{
Visual and electric spiking responses of seven types of rabbit retinal ganglion cells
}

\author{
Paul Werginz, Member, IEEE, Maesoon Im, Member, IEEE, Alex E. Hadjinicolaou, and Shelley I. \\ Fried, Member, IEEE
}

\begin{abstract}
Electric stimulation of the retina via retinal implants is currently the only commercially available method to restore vision in patients suffering from a wide range of outer retinal degenerations. To improve the quality of retinal implants, it is desirable to better understand how different retinal cell classes and types respond to electric stimuli so that more effective stimulation strategies can be developed. Here, we measured the response of seven major types of retinal ganglion cells to electric stimulation. A simple series of light stimuli were used to classify cells into known types. Electric stimulation produced unique responses in almost all ganglion cell types and the electric responses typically matched elements of the corresponding light responses.
\end{abstract}

\section{INTRODUCTION}

Multiple studies have reported a vast diversity of retinal ganglion cell (RGC) types in the mammalian retina [1-4]. Each type exhibits a characteristic response to light stimulation (LS) and transmits its distinct signal to a different portion of the brain.

We have shown previously that the responses to electric stimulation (ES) are also distinct in a few RGC types [5,6], and further, that some of the ES response properties match the corresponding LS response properties in these types [6]. It is still unknown however whether the majority of RGC types have unique responses to ES and if so, whether such responses also match their corresponding LS responses.

\section{METHODS}

The care and use of animals followed all federal and institutional guidelines and all protocols were approved by the Institutional Animal Care and Use Committee (IACUC)

*Research was supported by the Austrian Science Fund (FWF, J3947), by the VA (RR\&D: RX001663) and NIH (U01-NS099700). All authors were with the Department of Neurosurgery, Massachusetts General Hospital, Harvard Medical School, Boston, MA 02114 USA at the time of this study

P. Werginz is also with the Institute for Analysis and Scientific Computing, Vienna University of Technology, 1040 Vienna, Austria (email: werginz.paul@mgh.harvard.edu).

M. Im is now with the Department of Ophthalmology, Henry Ford Health System, the Department of Anatomy and Cell Biology, Wayne State University School of Medicine, and the Department of Electrical and Computer Engineering, Wayne State University College of Engineering, Detroit, MI 48202 USA (e-mail: mim1@hfhs.org).

A. E. Hadjinicolaou is now with the Department of Neurology, Massachusetts General Hospital, Harvard Medical School, Boston, MA 02114 USA (e-mail: ahadjinicolaou@mgh.harvard.edu).

S. I. Fried is also with the Boston VA Healthcare System, Rehabilitation, Research and Development, Boston, MA 01230 USA (e-mail: fried.shelley@mgh.harvard.edu). of the Massachusetts General Hospital. New Zealand White rabbits (Charles River Laboratories, Wilmington, MA, USA, $\sim 2 \mathrm{~kg}$ ) were anaesthetized with a mix of ketamine (75 $\mathrm{mg} / \mathrm{kg})$ and xylazine $(10 \mathrm{mg} / \mathrm{kg})$. Animals were subsequently euthanized by an intracardial injection of euthasol and eyeballs were harvested. After hemisection, the front portion of the eye and the lens were removed and any remaining vitreous eliminated. The retina was separated from the sclera and pigment epithelium and mounted, photoreceptor side down, onto a recording chamber. The retina was subsequently perfused with oxygenated Ames medium (Sigma-Aldrich, St. Louis, MO, USA) at a flow rate of 2-3 $\mathrm{ml} / \mathrm{min}$ for the duration of the experiment. Temperature was kept at $\sim 34{ }^{\circ} \mathrm{C}$. Targeted cells were located approximately $3-5 \mathrm{~mm}$ inferior to the visual streak. Small holes were made in the inner limiting membrane in order to access the RGC somata. Spiking responses to light and electric stimuli were obtained using loose (cell-attached) patch recordings. Patch electrode resistance was 8-14 M 2 .

Visual stimulation consisted of bright or dark spots with diameters ranging from $100-1000 \mu \mathrm{m}$ and presented for 1 sec. Only cells that exhibited consistent ON or OFF responses across the full range of spot sizes were included. Also, cells with sluggish responses [1] were excluded from further analysis.

ES was delivered by a $10 \mathrm{k} \Omega$ electrode (Micro-Probes, Gaithersburg, MD, USA) located $\sim 25 \mu \mathrm{m}$ above the inner limiting membrane and centered over the soma. The stimulus was a 4 ms cathodic monophasic half-sinusoid with a peak amplitude of $80 \mu \mathrm{A}$. Stimuli were delivered by a stimulus generator (STG 2004, Multi-Channel Systems MCS $\mathrm{GmbH}$, Reutlingen, Germany).

Stimulus control and data acquisition were performed with custom software written in LabView (National Instruments, Austin, TX, USA) and Matlab (Mathworks, Natick, MA, USA). Data were recorded using an Axopatch 200B amplifier (Molecular Devices, Sunnyvale, CA, USA) and digitized by a data acquisition card (PCI-MIO-16E-4, National Instruments, Austin, TX, USA). The timing of individual spikes was detected as the depolarization (negative) peak of each spike in the raw trace. Peri-stimulus time histograms (PSTH) were computed by averaging binned spikes from several trials $(\geq 3$ for visual stimulation and $\geq 6$ for electric stimulation). Bin size was $20 \mathrm{~ms}$ for visual stimulation and $5 \mathrm{~ms}$ for electric stimulation. 
A1
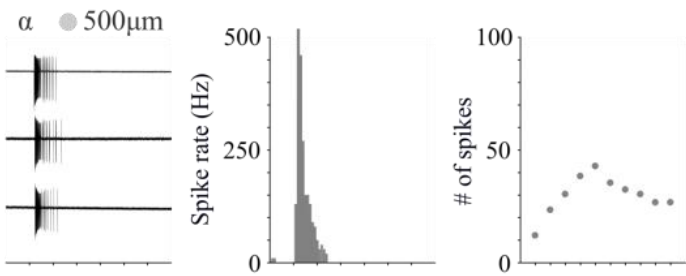

A2
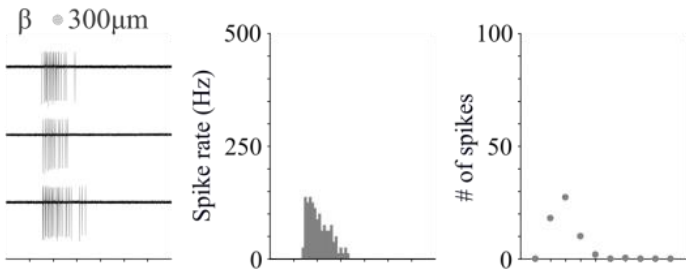

A3
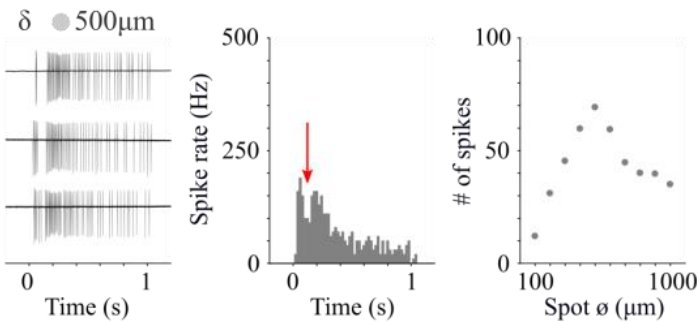

B1
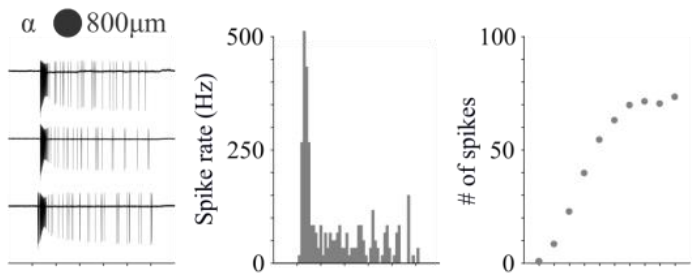

B2
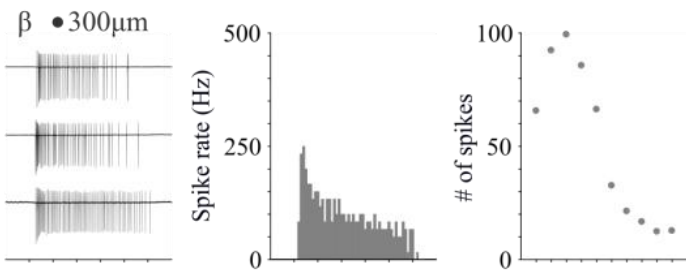

B3
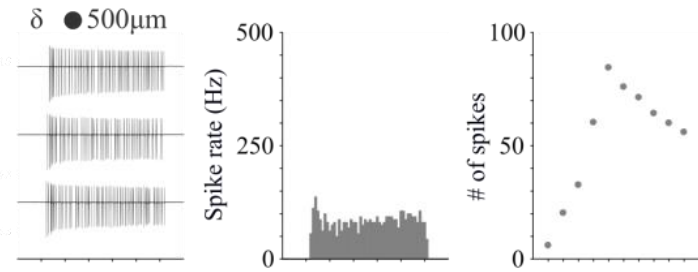

B4
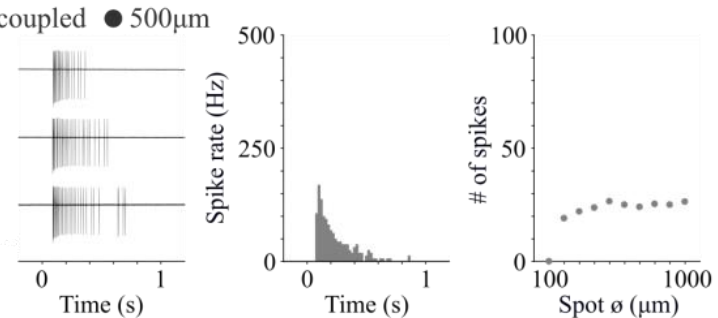

Figure 1. Spiking responses of one sample cell for three ON (A) and four OFF (B) RGC types to light stimuli. For each RGC type three raw recordings (left), PSTH (middle, bin width $20 \mathrm{~ms}$ ) and the number of elicited spikes for different spot diameters (right) are shown. Cell type and optimal spot size are indicated on top of each panel. See text for details.

\section{RESULTS}

Seven different types of RGCs $(\mathrm{ON}-\alpha, \mathrm{ON}-\beta, \mathrm{ON}-\delta, \mathrm{OFF}-$ $\alpha$, OFF- $\beta$, OFF- $\delta$ and OFF-coupled) were identified by their response to light (Fig. 1, Table 1). This corresponds to almost all of the RGC types identified in previous studies of the rabbit retina (see Conclusion) and we use the naming scheme of Roska et al. [4] for consistency. In total, we recorded from $26 \mathrm{ON}(\alpha=11, \beta=7, \delta=8)$ and $34 \mathrm{OFF}(\alpha=13$, $\beta=7, \delta=8$, coupled $=6$ ) cells.

\section{A. Visual stimulation}

ON cells could readily be distinguished into three classes by their responses to stationary flashes of bright spots varying in size from 100-1000 $\mu \mathrm{m}$. ON- $\alpha$ cells (Fig. 1 A1, brisk transient) responded with a $200-400 \mathrm{~ms}$ burst of spikes having a peak frequency of $\sim 500 \mathrm{~Hz}$. The response to very large spot sizes was not much weaker than that from medium spot sizes suggesting the strength of lateral inhibition to the cell was limited. ON- $\beta$ cells (Fig. 1 A2, brisk sustained) responded for a longer period of time and did not exhibit a transient peak in their PSTH. Greatly reduced spiking responses for larger spot sizes suggested a strong inhibitory surround for this cell type. $\mathrm{ON}-\delta$ cells (Fig. 1 A3) had a sustained response to the $1 \mathrm{sec}$ stimulus with a distinct 'dip' in the PSTH between the initial peak and the subsequent sustained component ([4], red arrow in Fig. 1 A3, middle). The strength of the inhibitory surround for this type was between that of the other two ON types.

Previous reports suggest an asymmetry in the number of ON and OFF RGC types [4] and that was confirmed here. Three of the four OFF cell types had sustained responses suggesting the OFF system may be more sustained than that of the ON. OFF- $\alpha$ cells (Fig. 1 B1, brisk transient) exhibited a strong spiking response but only for the first $\sim 100 \mathrm{~ms}$; the response was much weaker for the duration of the stimulus. These cells had the largest somata and a distinct 'triangular' shape which facilitated visual identification. Furthermore, the inhibitory surround was very weak for these cells. OFF- $\beta$ cells (Fig. 1 B2, brisk sustained) responded similarly to ON$\beta$ cells (opposite light polarity) but their responses were more sustained [7]. After an initial peak the response remained limited for the duration of the stimulus. Responses were strongly reduced for large spot sizes, again suggesting a strong inhibitory surround in the $\beta$ system. The OFF- $\delta$ class (Fig. 1 B3) responded with sustained spiking but without a pronounced transient peak. In contrast to OFF- $\beta$ cells, individual spikes tended to cluster into mini 'bursts' (not shown), which helped to further distinguish between these two cell types. The inhibitory surround was also weaker for OFF- $\delta$ cells. OFF-coupled cells (Fig. 1 B4) exhibited a transient response with low peak spike rate $(\sim 200 \mathrm{~Hz})$. Lateral inhibition for this cell type was fairly weak.

These results suggest that the use of a relatively simple series of light stimuli allows unequivocal identification of 
TABLE I. RESPONSE PROPERTIES DURING VISUAL AND ELECTRIC STIMULATION

\begin{tabular}{c|c|c|c|c|c|c}
\hline & \multicolumn{5}{c|}{ Visual } & \multicolumn{3}{c}{ Electric } \\
\hline Type & RESP & $\begin{array}{c}\text { Opt. spot } \\
\text { size }(\boldsymbol{\mu} \text { m) }\end{array}$ & $\begin{array}{c}\text { Inhib. } \\
\text { surround }\end{array}$ & $\begin{array}{c}\# \text { of } \\
\text { bursts }\end{array}$ & $\begin{array}{c}\text { Peak firing } \\
\text { rate (Hz) }\end{array}$ & Onset (ms) \\
\hline ON- $\alpha$ & $\mathrm{t}$ & $500-600$ & $\begin{array}{c}\text { weak to } \\
\text { medium }\end{array}$ & $3-5$ & $400-600$ & $40-60$ \\
\hline ON- $\beta$ & $\mathrm{t}^{*}$ & $200-300$ & strong & 2 & $<200$ & $150-200$ \\
\hline ON- $\delta$ & $\mathrm{s}$ & $500-600$ & medium & $2-3$ & $<200$ & $>200$ \\
\hline OFF- $\alpha$ & $\mathrm{s}$ & $600-800$ & weak & $2-3$ & $600-800$ & 10 \\
\hline OFF- $\beta$ & $\mathrm{s}$ & $200-300$ & strong & 2 & $300-500$ & 10 \\
\hline OFF $-\delta$ & $\mathrm{s}$ & $500-600$ & $\begin{array}{c}\text { weak to } \\
\text { medium }\end{array}$ & $2-3$ & $300-500$ & 15 \\
\hline $\begin{array}{c}\text { OFF- } \\
\text { coupled }\end{array}$ & $\mathrm{t}$ & $400-500$ & weak & 2 & $500-700$ & 10 \\
\hline
\end{tabular}

the cell type in all of the most prominent RGCs. This approach is attractive because it allows much easier and faster classification of cells than previously described methods. In addition, the testing can be done as a prelude to further physiological investigation, e.g. cell identification does not require fixation with subsequent immunochemical processing.

\section{B. Electric stimulation}

The spiking responses to ES were also largely distinct for each cell type (Fig. 2, Table 1). In general, responses in the ON system exhibited greater variability between types than that of the OFF system. Although responses in all 4 types of OFF cells were generally similar in appearance, there were subtle differences in peak firing rate, burst duration, number of elicited spikes and/or onset latency across types (see below). The electric responses in Fig. 2 were obtained from the same cells as those used in Fig. 1.

$\mathrm{ON}-\alpha$ cells (Fig. 2 A1) responded with $\geq 3$ bursts and spike rate gradually declined for later bursts. The peak firing rate of the main network response (i.e. $2^{\text {nd }}$ and subsequent bursts, indicated in black in the PSTH, [6]) was similar to that of visual stimulation $(\sim 400-600 \mathrm{~Hz})$ and was significantly higher than that of the two other ON RGC types. The onset of the network response in ON- $\beta$ cells (Fig. 2 A2) was significantly later (>150 ms) than that for ON- $\alpha$ cells. The peak firing rate was approximately $200 \mathrm{~Hz}$. The response patterns of $\mathrm{ON}-\alpha$ and $\mathrm{ON}-\beta$ cells are consistent with those of ON BT and BS cells reported in our previous work [6]. The network response of $\mathrm{ON}-\delta$ cells (Fig. 2 A3) had a peak frequency $<200 \mathrm{~Hz}$ and its onset was delayed (> $200 \mathrm{~ms}$ ) relative to that of the other ON types. An additional feature observed in $\mathrm{ON}-\delta$ cell responses was the presence of a mini-burst (1-3 spikes) that often occurred with a latency of 100-150 ms after stimulus onset (red arrow in Fig. 2 A3, right).
A1
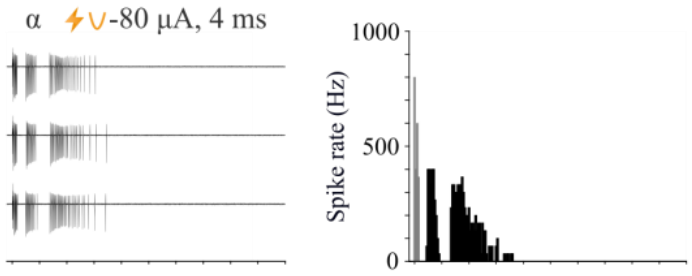

A2
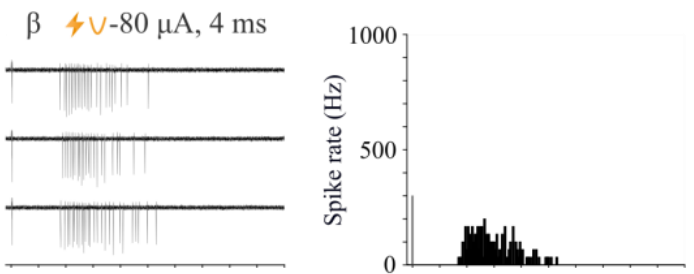

A3
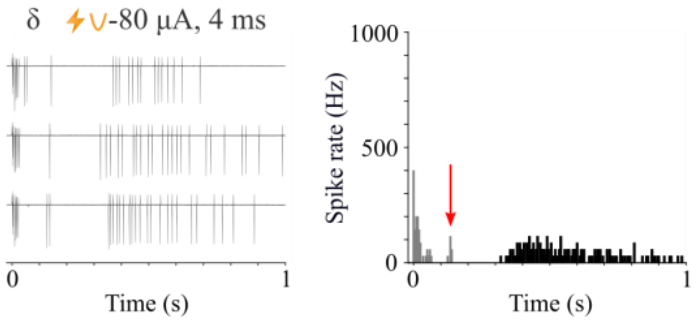

B1
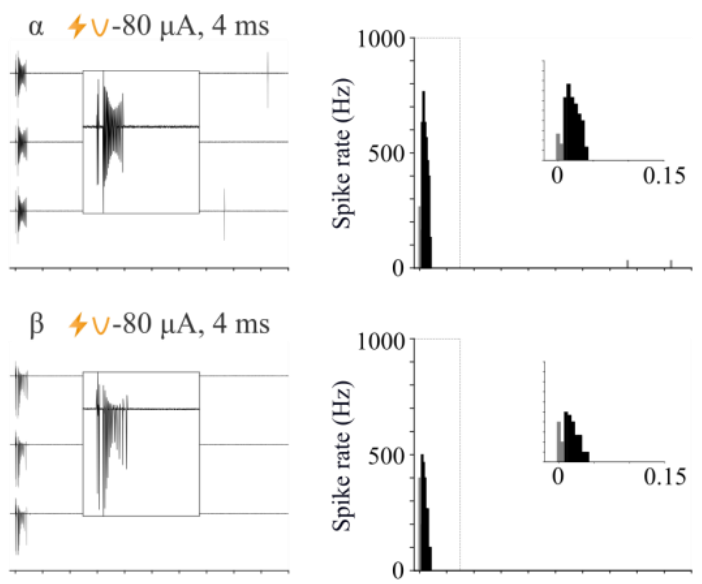

\section{B2}

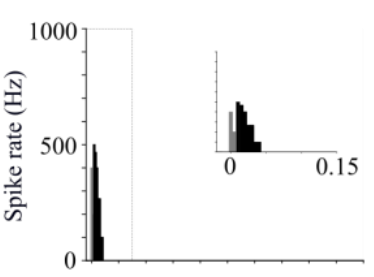

B3
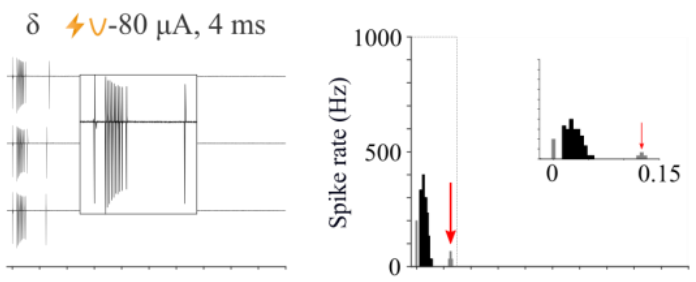

B4 coupled $4 \mathrm{~V}-80 \mu \mathrm{A}, 4 \mathrm{~ms}$
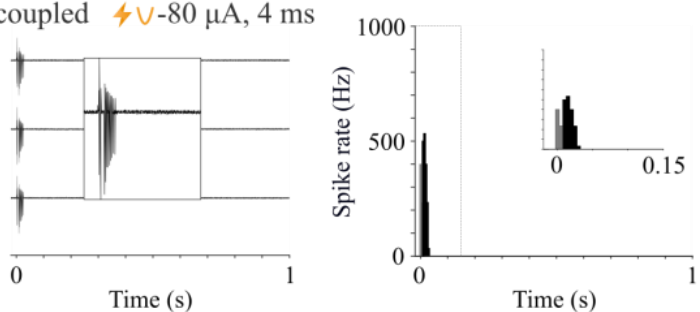

Figure 2 Spiking responses for three ON (A) and four OFF (B) RGC types to electric stimulation. The stimulus was a cathodic $4 \mathrm{~ms}$ monophasic halfsine wave with a maximum amplitude of $80 \mu \mathrm{A}$. Three raw recordings (left) and PSTH (right, bin width $5 \mathrm{~ms}$ ) are shown. Insets in OFF panels expand the first $150 \mathrm{~ms}$ of the response. See text for details. 
In contrast to the ON RGC responses, all OFF RGC types exhibited the same general response pattern to electric stimulation, namely 1-3 direct spikes followed by a slightly delayed, robust network response that persisted for 20-100 ms (Fig. 2 B). Analogous to the responses to visual stimuli, OFF- $\alpha$ cells (Fig. 2 B1) also responded to electric stimulation with the highest peak frequencies $(>600 \mathrm{~Hz})$. The expanded view of the top trace (inset) reveals a transient decrease and then increase in the amplitude of elicited spikes; this is similar to that which occurs with LS (Fig. 1 B1, left). OFF- $\beta$ cell responses (Fig. 2 B2) could be distinguished from $\mathrm{OFF}-\alpha$ cells by the lower peak spike rates $(\sim 300-500 \mathrm{~Hz})$. Note a similar transient decrease-increase in spike amplitude. The response of OFF- $\delta$ cells (Fig. 2 B3) had two distinct features that facilitate separation from other OFF cell types. i) Onset latency was significantly higher (>15 ms) than for the other three OFF RGC types and ii) a distinct additional mini-burst after approximately 100-150 ms could be observed (red arrow in Fig. 2 B3, right). OFFcoupled cells (Fig. 2 B4) had a short (20-30 ms), high frequency $(500-700 \mathrm{~Hz})$ response which resembles the response of OFF- $\beta$ cells. However, small differences in peak spike frequency and burst duration facilitated differentiation between these cell types. Further, the transient decreaseincrease in spike amplitude observed consistently in OFF- $\beta$ cells was never observed in OFF-coupled cells.

\section{CONCLUSION}

We did not find the parasol cell of Roska et al. [4] and speculate that it was one of the types we avoided, e.g. either its response to the simple light flashes used here was sluggish, or, it exhibits ON-OFF responses to one or more spot sizes. Our findings suggest that most of the rabbit RGC types can be readily identified by a simple LS protocol, at least for non-sluggish cells. Furthermore, we show that ES generates unique responses in all recorded RGC types (responses in OFF- $\beta$ \& OFF-coupled are somewhat similar but could still be distinguished). While here we used a relatively simple monophasic sinusoidal stimulus to characterize electric responses, it is likely that more complex stimuli may lead to larger differences between types, possibly because of differences in the calcium channel kinetics of presynaptic bipolar cells that presumably get activated by this stimulus [8]. The ability to readily identify cell types by LS as described here will help to facilitate this future work.

The two RGC types that exhibited rather similar electrical responses are OFF- $\beta$ \& OFF-coupled cells (Figure 2 B2 \& B4). This finding is somewhat surprising when comparing the respective light responses of the two types (Figure 1 B2 $\&$ B4). Whereas OFF- $\beta$ cells respond to light with a sustained spiking response that declines steadily throughout the course of the stimulus, OFF-coupled cells exhibit a rather short-duration high-frequency burst. Moreover, responses in OFF- $\beta$ cells are strongly suppressed for larger spot sizes whereas the inhibitory surround in OFF-coupled cells is much weaker. The strong similarities in ES therefore suggest that at least some portion of the electrical responses is shaped by different mechanisms than those that shape light responses. For example, the synaptic mechanisms that mediate lateral inhibition seem to not play much of a role during ES, at least not under the conditions used here. Identification of these methods will require additional studies.

\section{ACKNOWLEDGMENT}

We would like to thank Seung Woo Lee, Sang Baek Ryu and Vineeth Raghuram for helpful discussions during the preparation of the manuscript.

\section{REFERENCES}

[1] J. H. Caldwell and N. W. Daw, "New properties of rabbit retinal ganglion cells", J. Physiol., vol. 276, pp. 257-276, 1978.

[2] F. R. Amthor, E. S. Takahashi and W. Oyster, "Morphologies of rabbit retinal ganglion cells with complex receptive fields", J. Comp. Neurol., vol 280, pp. 97-121, 1989.

[3] R. L. Rockhill, F. J. Daly, M. A. MacNeil, S. P. Brown and R. H. Masland, "The diversity of ganglion cells in a mammalian retina", $J$. Neurosci., vol 22, pp. 3831-3843, 2002.

[4] B. Roska, A. Molnar and F. Werblin, "Parallel processing in retinal ganglion cells: How integration of space-time patterns of excitation and inhibition form the spiking output", J. Neurophysiol., vol. 95, pp. 3810-3822, 2006.

[5] S. W. Lee, D. K. Eddington and S. I. Fried, "Responses to pulsatile subretinal electric stimulation: effects of amplitude and duration", $J$. Neurophysiol., vol. 109, pp. 1954-1968, 2013.

[6] M. Im and S. I. Fried, "Indirect activation elicits strong correlations between light and electric responses in ON but not OFF retinal ganglion cells", J. Physiol., vol. 593, pp. 3577-3596, 2015.

[7] I. Buldyrev and W. R. Taylor, "Inhibitory mechanisms that generate centre and surround properties in ON and OFF brisk-sustained ganglion cells in the rabbit retina", J. Physiol., vol. 591, pp. 303-325, 2013.

[8] D. K. Freeman, J. S. Jeng, S. K. Kelly, E. Hartveit and S. I. Fried, "Calcium channel dynamics limit synaptic release in response to prosthetic stimulation with sinusoidal waveforms", J. Neural Eng., vol. 8, 046005, 2011.

(C) 2018 IEEE. Personal use of this material is permitted. Permission from IEEE must be obtained for all other uses, in any current or future media, including reprinting/republishing this material for advertising or promotional purposes, creating new collective works, for resale or redistribution to servers or lists, or reuse of any copyrighted component of this work in other works. 